\title{
A Switching Control Law for a Networked Visual Servo Control System
}

\author{
Haiyan $\mathrm{Wu}^{1}$, Chih-Chung Chen ${ }^{1}$, Jiayun Feng ${ }^{1}$, \\ Kolja Kühnlenz ${ }^{12}$ and Sandra Hirche ${ }^{1}$ \\ ${ }^{1}$ Institute of Automatic Control Engineering (LSR) \\ ${ }^{2}$ Institute for Advanced Study (IAS) \\ Technische Universität München \\ D-80290 München, Germany
}

Email: \{haiyan.wu, chen, koku, hirche\}@tum.de, jiayun.feng@mytum.de

\begin{abstract}
In this paper, a novel switching controller is proposed for a networked visual servo control system with varying feedback delay due to image processing and data transmission. The varying image processing delay caused by the varying number of extracted features for pose estimation due to different view angles, illumination conditions and noise, is modeled by its occurrence probability. The time delay due to transmission over the communication network is also modeled as random process. By using a sampled-data system approach and an input-delay approach, the linearized visual servo control system is reformulated into a stochastic continuous-time system with time-varying delay. A novel stability condition and associated switching controller are derived based on the occurrence probabilities of delays. Experiments on a 1-DoF linear module equipped with a camera are conducted to validate the proposed approach. A non-switching controller approach is implemented for comparison. The experimental results demonstrate significant performance improvement of the proposed control approach.
\end{abstract}

\section{INTRODUCTION}

It has long been recognized that the integration of vision into control is fundamental to increase the accuracy, versatility and application domain of robots in manufacturing. The use of visual-data in the feedback control loop is referred to as visual servo control, see [1] for an overview on advantages and challenges of visual servo control systems. With recent advances in communication and computing technologies, video grabbing, image processing and control can be implemented on different platforms across a common communication network. This kind of setup results in networked visual servo control systems (NVSCSs), see Fig. 1 for a visualization. The benefits of an NVSCS include: an NVSCS employs different cameras over a network; it provides wide-range visual feedback and increases system autonomy. An NVSCS may employ distributed computation for image processing; it enables high-speed vision feedback and is more robust to occlusions, see [2] for details.

However, using visual-data in the feedback loop causes delay from image acquisition, image processing and data transmission [3], [4]. In particular, time required for image processing depends not only on the platform, on which the algorithm is implemented, but also on the selection of image processing algorithm, which is designed for a specific application. For position-based visual servoing (PBVS) con-

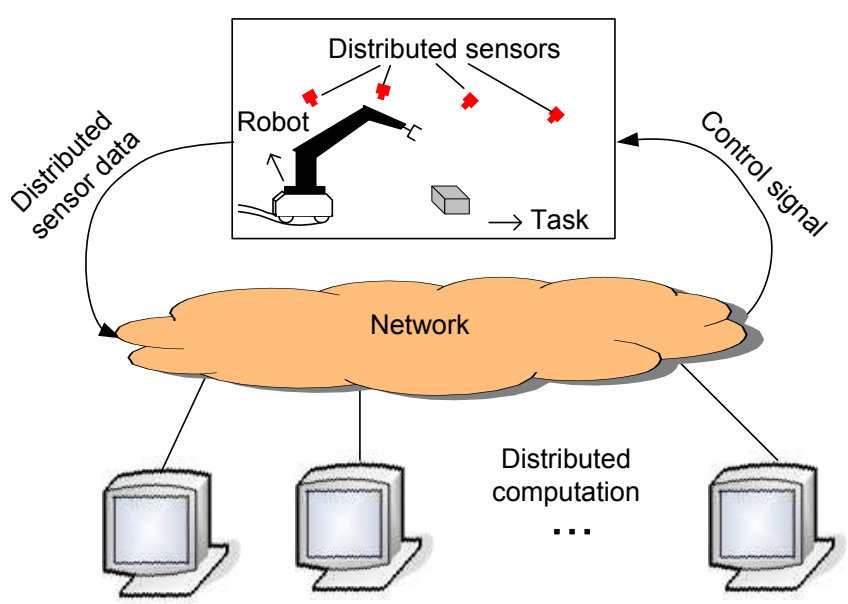

Fig. 1: Scheme of networked visual servo control systems with distributed sensors and distributed computations.

sidered in this paper, features are extracted from the image and used to estimate the pose of the target with respect to the camera. Therefore, the delay of image processing includes the time required for feature extraction, feature matching and pose estimation in PBVS. Furthermore, exchanging visualdata over a communication network is affected by random time delay and possibly packet loss. In this paper we consider systems with random time delay. Note that packet loss can be viewed as fictitious delay [5] and can therefore be also considered within the presented formulation.

Time delay is known to be a source of instability and deteriorates the control performance. In [6], [7], the performance limitation due to feedback delay has been addressed. However, system stability analysis and controller design regarding the feedback delay are not considered. For applications where more complex image processing algorithms are demanded, longer computational delay is inevitable and should be taken into consideration for system analysis. In the past literature, various methodologies have been developed to handle systems with delay [8]-[14]. In [8]-[10], systems with deterministic delay are studied by using standard analysis approaches from time-delay systems or robust control. 
However, these stability results can only be derived for worstcase delay and discard probability distributions of delays. This results in conservative controller design for NVSCSs.

A less conservative controller design approach refers to the switching of controllers according to delays, see [11][14] for details and technical realization. The random delay is modeled by a Markov process. Systems with delaydependent switching controller result in Markovian jump linear systems (MJLSs). The stability conditions are derived by Lyapunov methods. Associated delay-dependent controllers are designed by solving linear matrix inequality (LMI) problems with known probability transition rates of delays. However, the camera takes an image whenever the processing of the previous image is finished in our specific setting. Hence, the sampling interval is equal to the computational delay and has the same stochastic properties. The resulting stochastic delay model has the holding time correlated to its state, i.e. the sampling intervals. In this case, the Markovian modeling is no more applicable.In our approach, a set of indicator functions having independent identical distribution (i.i.d.) is introduced to model the delays.

In this paper, a novel switching controller is proposed for a networked visual servo control system with varying feedback delay. The numbers of image features vary from frame to frame due to different viewing angles, illumination conditions and noise. The time required for image processing in PBVS is thus modeled as a random process. As a simplification, we consider a local linear approximation of a visual servo control system. An analysis and design approach is proposed for this special case. A visual servo control system with sensor-to-controller random delay is reformulated into a stochastic continuous-time system with time-varying delay. An LMI-based delay-dependent stability condition is derived by a Lyapunov-Krasovskii functional and the associated delay-dependent switching controller design algorithm is presented in terms of LMI. The stability condition and controller design algorithm are determined based on occurrence probabilities of delays. The proposed approach is experimentally validated in a visual servo system comprising a pair of 1-DoF linear motor modules, one equipped with a camera. As benchmark a conventional nonswitching approach is implemented for comparison. The experimental results demonstrate a significant performance improvement by the proposed approach.

The remainder of the paper is organized as follows: The problem statement of visual servo sampled-data control is given in Section II. In Section III, a stochastic continuoustime system with time-varying delays is introduced by using input-delay approach. An LMI controller design algorithm is established in Section IV. In Section V, the experimental validation and performance comparison are discussed. This paper is concluded in Section VI.

Notation. In this paper $\lambda_{\max }(M)$ and $\lambda_{\min }(M)$ denote the maximal and the minimal eigenvalues of a matrix $M$, whereas $M^{T}$ and $\|M\|$ denote the transpose operator and induced Euclidean norm of matrix (or vector) $M$, respectively. The symbol $*$ denotes the transpose of the blocks outside the

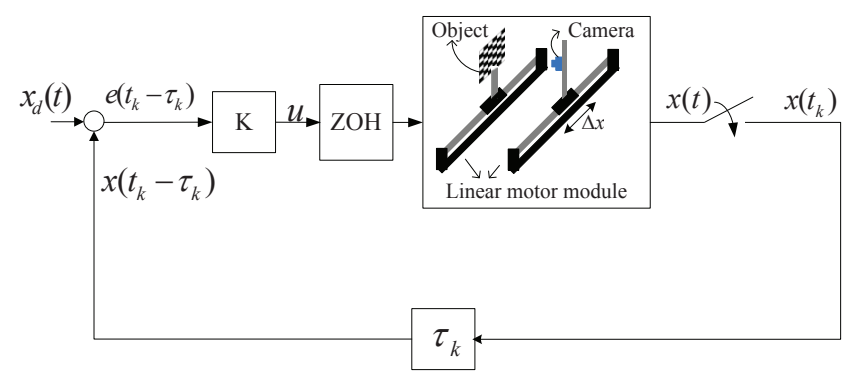

Fig. 2: Scheme of the visual servo control system with linear motor modules, one equipped with a camera.

main diagonal block in symmetric matrices. $\mathbb{E}$ stands for mathematical expectation and Pr for probability.

\section{PROBLEM SETTING}

In this paper, a tracking problem with a simple networked visual servo control is studied. A camera-in-hand structure is selected for tracking of a moving target with PBVS, as shown in Fig. 2.

For image processing, image features are firstly extracted from the image sequence. Then the pose of the target with respect to the camera is estimated. The controller is designed to reduce the error between the current pose $x_{c}$ and desired pose $x_{d}$. Time delay for computing the pose from visual data, which consists of time for image capturing, feature extraction, feature matching and pose estimation, is inevitable. Moreover, image features vary from frame to frame due to different view angles, illumination conditions and noise. This causes random delay for pose estimation which depends on the number of image features.

\section{A. Pose Estimation}

The fundamental issue of the tracking system with PBVS is pose estimation. By given matched feature pairs, the pose estimation problem could be considered as dual problem of $2 \mathrm{D}$ visual servoing proposed in [15]. A virtual camera is applied and is moved by using a visual servoing law to minimize the position errors between current observed image features and previous ones.

In order to increase the accuracy of pose estimation, Scale Invariant Feature Transform (SIFT) [16] which is known for its robust character is applied for feature extraction. To improve performance, SIFT is implemented on a GPU (Graphics Processing Units) exploiting its massive parallel processing capability. Matched feature pairs contain outliers, which lead to errors of the pose estimation. Therefore, RANSAC (RANdom Sample Consensus) [17] algorithm is used for the rejection of outliers.

\section{B. Visual servo control with feedback delay}

For computing efficiency, a distributed system structure is applied, by which image processing and manipulator control run in parallel on two PCs. The configuration of the manipulator is controlled through a host PC. The image processing is implemented on a standalone $\mathrm{PC}$ and its results, 


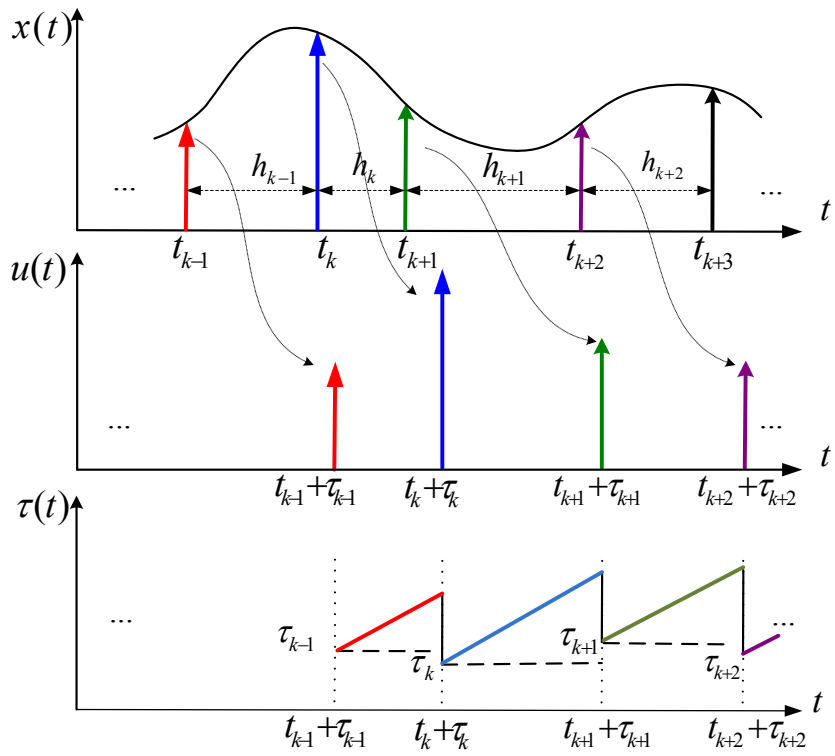

Fig. 3: Timing diagram of a networked visual servo control system, $\tau_{k}$ denotes the time-delays of image processing and data transmission.

i.e. difference between current and desired camera pose, are fed back through a communication network to the host PC.

The timing diagram of a networked visual servo control system is shown in Fig. 3, where $\tau_{k}$ is the time-delays caused by image processing and data transmission. At time $t_{k}$, processing of the current captured image starts. The extracted image features are fed into the pose estimation algorithm. As soon as pose estimation is finished, a new image is acquired and processed. The pose estimation results are transferred to the host PC through the communication network, and experience a random transmission delay. For the considered visual servo control system, the total delay, $\tau(t)$, contains mainly three parts: the image processing delay, the transmission delay and the sampling interval, as shown in Fig. 3.

For the preliminary study of an NCSCS with delays, we consider a linearized system as shown in Fig. 2. The system dynamics with identified parameters $m, c$ is given by

$$
\dot{x}(t)=A x(t)+B u(t),
$$

where

$$
A=\left[\begin{array}{cc}
0 & 1 \\
0 & -\frac{c}{m}
\end{array}\right] \text { and } B=\left[\begin{array}{cc}
0 & 0 \\
\frac{1}{m} & 0
\end{array}\right] .
$$

Consider a controller $u(t)=K x\left(t_{k}\right)$ with $t_{k}$ representing the sampling instant. The closed-loop system is derived as

$$
\dot{x}(t)=A x(t)+B K x\left(t_{k}\right), \quad t_{k} \leq t<t_{k+1} .
$$

Remark 1: Assuming a more general manipulator dynamics $M(q) \ddot{q}+C(q, \dot{q})=\Gamma$ and a Jacobian transpose controller $\Gamma=J^{T} K x$, a general closed-loop formulation of PBVS is [18]

$$
\frac{d}{d t}\left[\begin{array}{c}
q \\
\dot{q}
\end{array}\right]=\left[\begin{array}{c}
\dot{q} \\
D
\end{array}\right]
$$

where

$$
D=M^{-1}(q)\left(J^{T}(q) K x(t)-C(q, \dot{q})\right),
$$

with $q$ joint displacements, $M(q)$ manipulator inertia matrix, $C(q, \dot{q})$ centripetal and Coriolis torques, $J(q)$ robot Jacobian. The existence of a stable equilibrium and regularity of the Jacobian in a sufficiently small region around this equilibrium are assumed for stability analysis according to [19]. The linearized model at the origin $q \equiv 0$ can be written as

$$
\frac{d}{d t}\left[\begin{array}{c}
q \\
\dot{q}
\end{array}\right]=\left[\begin{array}{cc}
0 & 1 \\
\frac{d}{d q} D & \frac{d}{d \dot{q}} D
\end{array}\right] .
$$

Thus, in case of the considered 1-DoF system with its dynamics $\Gamma=m \ddot{q}+c \dot{q}$, we arrive at the simplified model (1). The stability analysis and controller design algorithm proposed here can be applied to nonlinear visual servoing systems with more DoF locally by linearizing the system at equilibrium states or by the computed torque feedforward approach proposed in [20].

It is assumed that the delay of image processing and data transmission causes the sampled data $x\left(t_{k}\right)$ arriving at the controller with random delay $\tau_{k} \geq 0$, so the closed-loop system in (2) becomes

$$
\dot{x}(t)=A x(t)+B K x\left(t_{k}\right), \quad t_{k}+\tau_{k} \leq t<t_{k+1}+\tau_{k+1} .
$$

The closed-loop system in (3) has a non-deterministic sampling period

$$
h_{k}=t_{k+1}-t_{k}+\tau_{k+1}-\tau_{k},
$$

depending on delays of $k^{\text {th }}$ and $(k+1)^{t h}$ sampled measurement. Due to the randomness of $\tau_{k}$ and $\tau_{k+1}$, the sampling period $h_{k}$ is also random. The problem to be addressed in this paper is formulated as follows:

Problem Given a system in (3) with random sampling intervals $h_{k}$, develop a control algorithm such that the closedloop system in (3) is exponential mean-square stable (EMSS) satisfying

$$
\mathbb{E}\left\{\|x(t)\|^{2} \mid x\left(t_{0}\right)\right\} \leq b\left\|x\left(t_{0}\right)\right\|^{2} e^{-\rho\left(t-t_{0}\right)},
$$

where $b>0, \rho>0$ are real numbers and $x\left(t_{0}\right)$ is the initial condition.

\section{SYSTEM REFORMULATION BASED ON INPUT-DELAY APPROACH}

In this section, the NVSCS with aperiodic sampling is reformulated into a continuous-time system with time-varying delay by means of the input-delay approach. A random process is introduced to describe delays caused by image processing and network transmission. A delay-dependent switching controller is considered, finally resulting in a stochastic continuous-time system with time-varying delays. 


\section{A. Input-delay transformation}

Reconsider the sampling instant $t_{k}$ as

$$
\begin{aligned}
t_{k} & =t-\left(t-t_{k}\right) \\
& =t-\tau(t), \quad t_{k}+\tau_{k} \leq t<t_{k+1}+\tau_{k+1} .
\end{aligned}
$$

It is assumed that the overall time-delay is bounded by

$$
\begin{aligned}
\bar{\tau} & =\min _{k \in \mathbb{K}}\left\{\tau_{k}\right\}, \\
\underline{\tau} & =\max _{k \in \mathbb{K}}\left\{t_{k+1}-t_{k}+\tau_{k+1}\right\} .
\end{aligned}
$$

The closed-loop system in (3) is reformulated into a continuous-time system with time-delay

$$
\begin{gathered}
\dot{x}(t)=A x(t)+B K x(t-\tau(t)), \\
x_{0}=x(t+\theta), \quad \theta \in[-\bar{\tau}, 0], \\
t \in\left[t_{k}+\tau_{k}, t_{k+1}+\tau_{k+1}\right) .
\end{gathered}
$$

Remark 2: The reformulation of a sampled-data system into a continuous-time system with time-varying delay is called input-delay approach. This approach was first introduced in [9] [21], where system periodic sampling is dealt with. Sampled-data systems with aperiodic (random) sampling using input-delay approach are addressed in [22]. However, no switching controller is considered. This paper further extends the concept derived in [22] to cope with NVSCSs with random delays.

\section{B. Stochastic system with time-varying delays}

For further technical development, the time-delay in the closed-loop system (3) is categorized into $n \geq 2$ intervals by $s_{i}>0, i=1, \ldots, n-1$, satisfying $s_{i}<s_{i+1}, s_{0}=\underline{\tau}$ and $s_{n}=\bar{\tau}$. The $n$ interval delays are defined as

$$
\begin{aligned}
\tau_{1}(t) & =\left\{\tau(t) \mid s_{0} \leq \tau(t)<s_{1}\right\}, \\
\tau_{2}(t) & =\left\{\tau(t) \mid s_{1} \leq \tau(t)<s_{2}\right\}, \\
& \vdots \\
\tau_{n}(t) & =\left\{\tau(t) \mid s_{n-1} \leq \tau(t)<s_{n}\right\} .
\end{aligned}
$$

The occurrence of $\tau_{i}(t), i=1, \ldots, n$, is described by a set of indicator functions

$$
\beta_{i}(t)= \begin{cases}1, & s_{i-1} \leq \tau(t)<s_{i}, \quad i=1, \ldots, n \\ 0, & \text { otherwise }\end{cases}
$$

satisfying

$$
\operatorname{Pr}\left\{\beta_{i}(t)=1\right\}=p_{i}, \quad \sum_{j=1}^{n} p_{i}=1 .
$$

The expected value and variation of $\beta_{i}(t)$ are given by

$$
\mathbb{E}\left\{\beta_{i}(t)\right\}=p_{i}, \quad \mathbb{E}\left\{\left(\beta_{i}(t)-p_{i}\right)^{2}\right\}=p_{i}\left(1-p_{i}\right) .
$$

In order to increase the control performance, a delaydependent switching controller, which switches its feedback gain according to the active delay interval, is introduced. The control law of the system in (3) becomes

$$
u(t)=K_{i} x\left(t-\tau_{i}(t)\right), \quad i=1, \ldots, n .
$$

According to (6)-(7), the closed-loop system in (3) can be rewritten as

$$
\dot{x}(t)=A x(t)+\sum_{i=1}^{n} \beta_{k}(t) B K_{i} x\left(t-\tau_{i}(t)\right) .
$$

Remark 3: Note that the feedback gain $K_{i}$ of system (8) is switched according to delays $\tau_{i}(t)$ and results in a randomly switched time-varying delay system. It is assumed that each switching of (8) is separated by a finite time interval. Therefore, the Zeno solutions are excluded in this paper.

\section{STABILITY ANALYSIS AND CONTROLLER DESIGN}

The objective of this section is to derive an exponential mean-square stability condition for NVSCSs in (8) and to design a delay dependent switching controller.

\section{A. Stability Analysis}

The Lyapunov-Krasovskii approach is considered to cope with the stability of the system (8), as it is stochastic and contains time-varying delays. Generally, the stability condition derived by Lyapunov-Krasovskii approach can be categorized into two types; a delay-independent and delay-dependent condition. In order to derive a delay-dependent condition, the following Newton-Leibnitz formula is considered

$$
\int_{t-\tau_{i}(t)}^{t} \dot{x}(s) d s=x(t)-x\left(t-\tau_{i}(t)\right), \quad i=1,2 .
$$

Substitute the Newton-Leibnitz formula into (8) and let $z^{T}(t)=\left[x^{T}(t) \dot{x}^{T}(t)\right]$. The closed-loop system (8) yields

$$
E \dot{z}(t)=\bar{A} z(t)-\sum_{i=1}^{n} \bar{A}_{i} \int_{t-\tau_{i}(t)}^{t} z(s) d s,
$$

where $E=\left[\begin{array}{ll}I & 0 \\ 0 & 0\end{array}\right]$,

$$
\bar{A}=\left[\begin{array}{cc}
0 & I \\
A+\sum_{i=1}^{n} \beta_{i}(t) B K_{i} & -I
\end{array}\right], \bar{A}_{i}=\left[\begin{array}{cc}
0 & 0 \\
0 & \beta_{i}(t) B K_{i}
\end{array}\right] .
$$

The system (9) is used for stability analysis. Details of the stability condition are given in the following theorem.

Theorem 1: For the closed-loop system in (8), if symmetric matrices exist, $Q_{1}>0, Q_{i}>0, i=1, \ldots, n, P_{1}>0$ and real matrices $P_{2}$ and $P_{3}$ with

$$
P=\left[\begin{array}{ll}
P_{1} & 0 \\
P_{2} & P_{3}
\end{array}\right]
$$

such that the following LMI is satisfied

$$
\left[\begin{array}{cccc}
\Psi & s_{1} P^{T} & \cdots & s_{n} P^{T} \\
* & -s_{1} Q_{1} & 0 & \vdots \\
\vdots & 0 & \ddots & * \\
* & \cdots & * & -s_{n} Q_{n}
\end{array}\right]<0
$$


where

$$
\begin{gathered}
\Psi=\left[\begin{array}{cc}
\Xi_{1} & \Xi_{2} \\
P_{1}-P_{2} & -P_{3}
\end{array}\right]+\left[\begin{array}{cc}
\Xi_{1} & \Xi_{2} \\
P_{1}-P_{2} & -P_{3}
\end{array}\right]^{T} \\
+\sum_{i=1}^{n} s_{i}\left[\begin{array}{cc}
0 & 0 \\
0 & p_{i} B K_{i}
\end{array}\right]^{T} Q_{i}\left[\begin{array}{cc}
0 & 0 \\
0 & p_{i} B K_{i}
\end{array}\right] \\
\Xi_{1}=A^{T} P_{2}+\sum_{i=1}^{n} p_{i}\left(B K_{i}\right)^{T} P_{2} \\
\Xi_{2}=A^{T} P_{3}+\sum_{i=1}^{n} p_{i}\left(B K_{i}\right)^{T} P_{3} .
\end{gathered}
$$
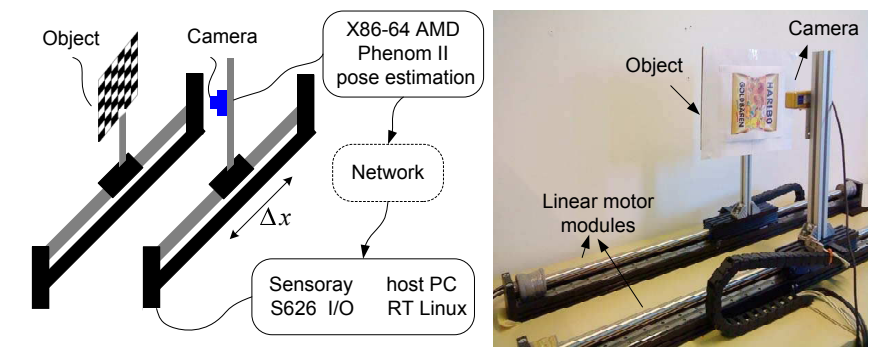

Fig. 4: Linear motor modules setup

then the trajectory of the system satisfies (4).

Proof: See Appendix 7.1.

The stability condition in Theorem 1 is derived in terms of an LMI, which can be efficiently solved by computational toolbox for Matlab, e.g. Yalmip [23].

Remark 4: Theorem 1 is derived by a similar Lyapunov candidate as used in Theorem 3 of [13]. However, the stability condition of [13] is determined by known probability transition rates of delays. This requires an exponential distribution of delay. Since the data processing delay is in general not exponentially distributed, the stability in Theorem 1 is conditioned merely by occurrence probabilities of random delays and the restriction on exponential distribution of delays is relaxed.

Remark 5: Note that due to the assumptions in Remark 1 only local stability is proven.

\section{B. Controller Design}

Solving feedback gains $K_{i}, i=1, \ldots, n$ in Theorem 1 involves nonlinear terms, e.g. $P_{2}^{T} B K_{1}, P_{3}^{T} B K_{1}, P_{2}^{T} B K_{2}$ and $P_{3}^{T} B K_{2}$ in (10). These nonlinear terms render the inequality in (10) into a bilinear matrix inequality (BMI) problem, whose solutions are difficult to find as it is non-convex and NP-hard. However, the nonlinear terms can be eliminated by choosing a special matrix $X=P^{-1}$ and so an LMI formulation is recovered. The controller design algorithm is given in the following theorem.

Theorem 2: Given positive scalars $r_{1}$ and $r_{2}$, if there exist symmetric matrices $R_{i}>0, i=1, \ldots, n$ and $X_{1}>0$ satisfying

$$
X=\left[\begin{array}{cc}
X_{1} & 0 \\
-r_{1} X_{1} & r_{2} X_{1}
\end{array}\right]
$$

such that

$$
\left[\begin{array}{cccc}
\hat{\Psi} & \hat{\Psi}_{1}^{T} & \cdots & \hat{\Psi}_{n}^{T} \\
* & -s_{1} R_{1} & 0 & \vdots \\
\vdots & 0 & \ddots & * \\
* & \cdots & * & -s_{n} R_{n}
\end{array}\right]<0
$$

where

$$
\begin{aligned}
\hat{\Psi}= & {\left[\begin{array}{cc}
-r_{1} X_{1} & r_{2} X_{1} \\
\Xi_{3} & -r_{2} X_{1}
\end{array}\right]+\left[\begin{array}{cc}
-r_{1} X_{1} & r_{2} X_{1} \\
\Xi_{3} & -r_{2} X_{1}
\end{array}\right]^{T} } \\
& +\sum_{i=1}^{n} s_{i} R_{i} \\
\Xi_{3}= & A X_{1}+\sum_{i=1}^{n} p_{i} B Y_{i}+r_{1} X_{1} \\
\hat{\Psi}_{1}= & s_{1} \bar{A}_{1} X=s_{1}\left[\begin{array}{cc}
0 & 0 \\
-p_{1} r_{1} B Y_{1} & p_{1} r_{2} B Y_{1}
\end{array}\right] \\
& \vdots \\
\hat{\Psi}_{n}= & s_{n} \bar{A}_{n} X=s_{n}\left[\begin{array}{cc}
0 & 0 \\
-p_{n} r_{1} B Y_{n} & p_{n} r_{2} B Y_{n}
\end{array}\right]
\end{aligned}
$$

holds, then the NVSCS is MES with the feedback gain

$$
K_{i}=Y_{i} X_{1}^{-1}, \quad i=1, \ldots, n .
$$

Proof: See Appendix 7.2.

Remark 6: The structure of matrix $X$ is chosen based on the requirement $P^{-1}=X$, where $E P=P^{T} E$. Therefore, $X$ is determined as follows

$$
X=\left[\begin{array}{cc}
X_{1} & 0 \\
X_{2} & X_{3}
\end{array}\right], \quad X_{1}=X_{1}^{T}>0 .
$$

However, by expanding $\hat{\Psi}_{i}, i=1, \ldots, n$ in (11) it results in terms, e.g. $B K_{i} X_{j}, i=1, \ldots, n$ and $j=1,2,3$, which make deriving an LMI formulation impossible. In order to obtain an LMI formulation, $X_{2}$ and $X_{3}$ in (13) are restricted to $-r_{1} X_{1}$ and $r_{2} X_{1}$, where $r_{1}$ and $r_{2}$ are positive real numbers.

Although LMI can be efficiently solved by LMI toolbox, the restriction on matrix $X$ introduces certain conservatism in the controller design and leads to unfeasibility in some cases. When the unfeasibility occurs, the general form of $\mathrm{X}$ in (13) is used and an BMI (bilinear matrix inequality) solver is applied to solve the problem in (11).

\section{EXPERIMENTS}

Experiments in this section are designed to validate the proposed control approach. The experimental testbed consists of two linear motor modules, as shown in Fig. 4. A target is mounted on the reference module, and the manipulator equipped with a camera is connected to the controlled module. Both modules are controlled through 
MATLAB/SIMULINK blocksets on host PC with the sampling period of $T=1 \mathrm{~ms}$. Standalone real-time code is generated directly from SIMULINK modules with the Realtime Workshop. The input signal assigned to the reference module is:

$$
x_{d}(t)=0.15 \sin (1.57 t) .
$$

The high-speed camera with a resolution of $640 \times 480$ pixels is connected to a second PC (X8664, AMD, Phenom II $\times 4810$ processor), which deals with image processing. As an approximation to an event-based system, a high image framerate of 400fps is used. In comparison with an ideal event-based approach, this results in a jitter of $2.5 \mathrm{~ms}$ with respect to the sampling rate of $1 \mathrm{kHz}$ of the robot controller. Compared to the time-delays of data transmission and image processing, it can be neglected and the system may thus be approximatively considered as an event-based system. The results of image processing are packetized with time-stamp and sent from second PC to host PC through network. The time required for realtime image processing depends on image features and has random values between $33 \mathrm{~ms}$ to $45 \mathrm{~ms}$. The relationship between image features and image processing delay is shown in Fig. 5 (a) (b). The cross correlation coefficient between these two signals is 0.9346, which indicates that image processing delay is closely related with image feature number. More image features require more time for image processing, e.g. 18 image features take about $33 \mathrm{~ms}$ while 55 image features take about $43.5 \mathrm{~ms}$. As soon as the image processing is finished, a new image is acquired and processed. This results in a random sampling intervals up to $45 \mathrm{~ms}$. In addition, random delay of data transmission is simulated by a network emulator (Netem) having i.i.d. delay ranging from $5 \mathrm{~ms}$ to $10 \mathrm{~ms}$. The overall delay (i.e. the sum of computation delay, transmission delay and sampling interval) has the range from $38 \mathrm{~ms}$ to $100 \mathrm{~ms}$ as shown in the Fig. 5 (c).

The dynamics of the linear motor module is identified as: $m=0.073, c=85.4$. According to (8) and considering $n=2$, the closed-loop system of the controlled module yields

$$
\begin{aligned}
\frac{d}{d t}\left[\begin{array}{l}
x(t) \\
\dot{x}(t)
\end{array}\right]= & {\left[\begin{array}{cc}
0 & 1 \\
0 & -1169.9
\end{array}\right]\left[\begin{array}{l}
x(t) \\
\dot{x}(t)
\end{array}\right] } \\
& +\beta(t)\left[\begin{array}{cc}
0 & 0 \\
K_{1} & 0
\end{array}\right]\left[\begin{array}{l}
x\left(t-\tau_{1}(t)\right) \\
\dot{x}\left(t-\tau_{1}(t)\right)
\end{array}\right] \\
& +(1-\beta(t))\left[\begin{array}{cc}
0 & 0 \\
K_{2} & 0
\end{array}\right]\left[\begin{array}{l}
x\left(t-\tau_{2}(t)\right) \\
\dot{x}\left(t-\tau_{2}(t)\right)
\end{array}\right] .
\end{aligned}
$$

$s_{1}=65 \mathrm{~ms}$ and $p=0.53$ are heuristically selected. Solving Theorem 2, the feedback gains are

$$
K_{1}=800, \quad K_{2}=160 .
$$

The experiment runs 20 times with the same initial conditions of both modules. For the comparison of control performance, the standard non-switching controller is implemented, i.e. a robust controller that stabilizes for all occurring time delays up to $\bar{\tau}=100 \mathrm{~ms}$. The corresponding controller

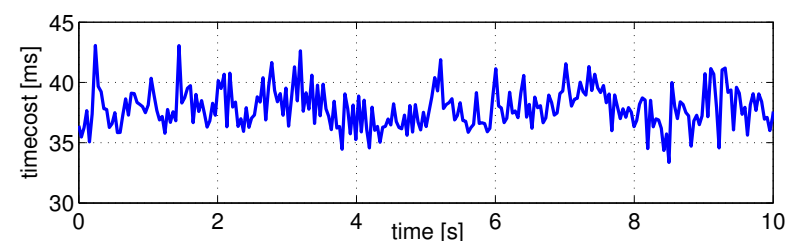

(a)

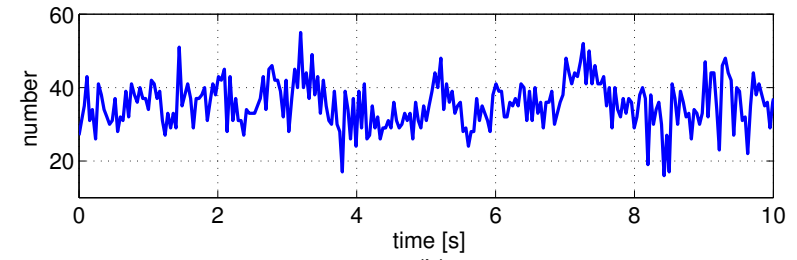

(b)

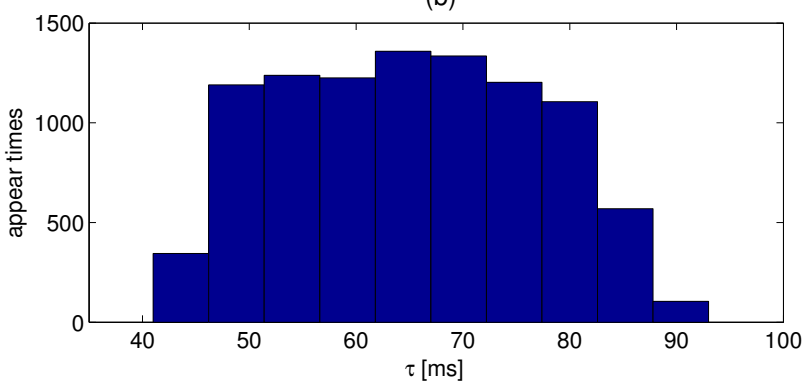

(c)

Fig. 5: Image processing time (a), extracted image features (b) and histogram of delays in the feedback loop (c).

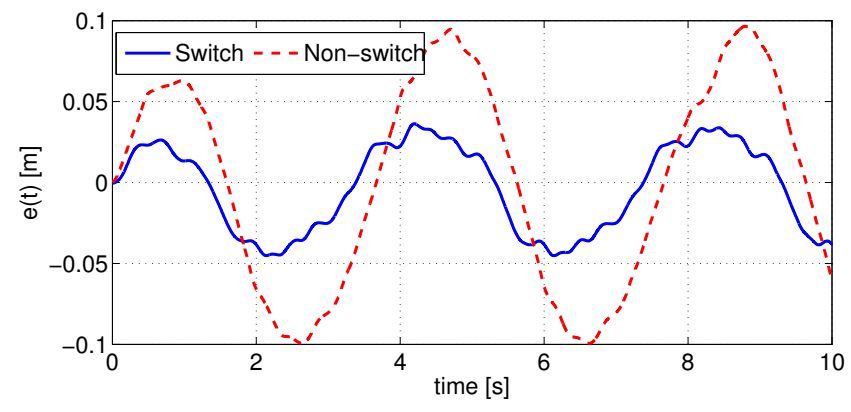

Fig. 6: Mean control error evolution of delay-dependent controller (solid line) standard design controller (dash line).

design gain is $K=160$. The control error is defined by

$$
e(t)=x_{d}(t)-x_{c}(t),
$$

where $x_{d}(t)$ and $x_{c}(t)$ denote the position measurements of the reference module and the controlled module respectively. The resulting control errors are shown in Fig. 6. The delay dependent switching controller approach has maximal tracking error $e_{\max }=3.63 \mathrm{~cm}$ and the variance of the tracking error $e_{\mathrm{var}}=7.22 \mathrm{~cm}^{2}$, while the maximal tracking error of standard design controller approach is $e_{\max }=9.66 \mathrm{~cm}$ and the variance of the tracking error $e_{\mathrm{var}}=42.95 \mathrm{~cm}^{2}$. The experimental results demonstrate that the switching controller approach leads to a better control performance compared to the conventional non-switching control design. 


\section{CONCLUSION}

This paper presents a novel analysis and design approach for networked visual servo control systems with random delays due to image processing and data transmission. The resulting system is a continuous-time system with stochastic variable and time-varying delays. The exponential meansquare stability is shown by using a Lyapunov-Krasovskii function. A delay-dependent controller is derived based on linear matrix inequalities (LMI). The proposed approach is validated in experiments. The results demonstrate performance benefits of the proposed design approach over the conventional counterpart. In the current stage, the approach is limited to visual servo control, which can be approximated by linear systems. Future work is concerned with extending the approach to more general nonlinear systems.

\section{APPENDIX}

Before the proof is shown, the following definition and lemma have to be given.

Definition 1: [24] Let $\mathscr{L}$ be the infinitesimal generator of a function $V(z(t))$. Then, the operator $\mathscr{L}$ acting on $V(z(t))$ is defined as

$$
\mathscr{L} V(z(t))=\lim _{\Delta \rightarrow 0} \frac{1}{\Delta}\{\mathbb{E}\{V(z(t+\Delta) \mid z(t))\}-V(z(t))\} .
$$

Lemma 1: [25] Let $X$ and $Y$ be real constant matrices with appropriate dimensions. Then

$$
X^{T} Y+Y^{T} X \leq \varepsilon X^{T} X+\frac{1}{\varepsilon} Y^{T} Y
$$

holds for any $\varepsilon>0$.

\section{A. Proof of Theorem 1}

Consider a Lyapunov candidate

$$
V(z(t))=V_{0}(z(t))+\sum_{i=1}^{n} V_{i}(z(t))
$$

where

$$
\begin{aligned}
& V_{0}(z(t))=z^{T}(t) E P z(t), \\
& V_{i}(z(t))=\int_{-s_{i}}^{0} \int_{t+\theta}^{t} z^{T}(s) \bar{A}_{i}^{T} Q_{i} \bar{A}_{i} z(s) d s d \theta .
\end{aligned}
$$

According to Definition 1, it has

$$
\begin{aligned}
\mathscr{L} V_{0}(z(t))= & \dot{z}^{T}(t) E P z(t)+z^{T}(t) P^{T} E \dot{z}(t) \\
= & z^{T}(t)\left[\bar{A}^{T} P+P^{T} \bar{A}\right] z(t) \\
& -2 \sum_{i=1}^{n} z^{T}(t) P^{T} \bar{A}_{i} \int_{t-\tau_{i}(t)}^{t} z(s) d s .
\end{aligned}
$$

According to Lemma 1, $\mathscr{L} V_{0}\left(z_{t}\right)$ becomes

$$
\begin{aligned}
\mathscr{L} V_{0}(z(t)) \leq & z^{T}(t)\left[\bar{A}^{T} P+P^{T} \bar{A}\right] z(t) \\
& +\sum_{i=1}^{n} s_{i} z^{T}(t) P^{T} Q_{i}^{-1} P z(t) \\
& +\sum_{i=1}^{n} \int_{t-s_{i}}^{t} z^{T}(s) \bar{A}_{i}^{T} Q_{i} \bar{A}_{i} z(s) d s .
\end{aligned}
$$

Likewise, it has

$$
\begin{aligned}
\sum_{i=1}^{n} \mathscr{L} V_{i}(z(t)) & =\sum_{i=1}^{n} s_{i} z^{T}(t) \bar{A}_{i}^{T} Q_{i} \bar{A}_{i} z(t) \\
& -\sum_{i=1}^{n} \int_{t-s_{i}}^{t} z^{T}(s) \bar{A}_{i}^{T} Q_{i} \bar{A}_{i} z(s) d s .
\end{aligned}
$$

Combine (15) and (16), it yields

$$
\begin{aligned}
\mathscr{L} V(z(t)) \leq & z^{T}(t)\left[\bar{A}^{T} P+P^{T} \bar{A}+\sum_{i=1}^{n} s_{i} \bar{A}_{i}^{T} Q_{i} \bar{A}_{i}\right. \\
& \left.+\sum_{i=1}^{n} s_{i} P^{T} Q_{i}^{-1} P\right] z(t) \\
= & z^{T}(t) \Theta z(t) .
\end{aligned}
$$

Apply Schur complement to (17), it results in (10).

Note that $\max _{\theta \in[-\tau, 0]}\{\|z(t+\theta)\|\} \leq \phi\|z(t)\|$ for some $\varphi>0$ [26], the following inequality can be established

$$
\begin{aligned}
V(z(t)) & \leq\left[\lambda_{\max }(E P)+\sum_{i=1}^{n} \frac{s_{i}^{2}}{2} \lambda_{\max }\left(Q_{i}\right)\right]\|z(t)\|^{2} \\
& \leq \Lambda_{\max }\|z(t)\|^{2} .
\end{aligned}
$$

Combining (17) and (18) yields

$$
\frac{\mathscr{L} V(z(t))}{V(z(t))} \leq-\frac{\lambda_{\min }(-\Theta)}{\Lambda_{\max }} \triangleq-\rho_{0}
$$

and

$$
\mathbb{E}\{\mathscr{L} V(z(t))\} \leq-\rho_{0} \mathbb{E}\{V(z(t))\} .
$$

By applying Dynkin's formula into (19) it becomes

$$
\begin{aligned}
& \mathbb{E}\{V(z(t))\}-\mathbb{E}\{V(z(0))\} \\
& =\mathbb{E}\left\{\int_{0}^{t} \mathscr{L} V(z(s)) d s\right\} \leq \\
& -\rho_{0} \int_{0}^{t} \mathbb{E}\{V(z(s))\} d s .
\end{aligned}
$$

Using the Gronwall-Bellman lemma, (20) results in

$$
\mathbb{E}\{V(z(t))\} \leq e^{-\rho_{0} t} \mathbb{E}\{V(z(0))\} .
$$

Since

$$
\begin{aligned}
V(z(t)) & \geq\left[\lambda_{\min }(E P)+\sum_{i=1}^{n} \frac{s_{i}^{2}}{2} \lambda_{\min }\left(Q_{i}\right)\right]\|z(t)\|^{2} \\
& =\Lambda_{\min }\|z(t)\|^{2}
\end{aligned}
$$

it is established that

$$
\mathbb{E}\left\{\|z(t)\|^{2}\right\} \leq e^{-\rho_{0} t} \frac{\mathbb{E}\{V(z(0))\}}{\Lambda_{\min }} .
$$

Equation (21) provides the proof for mean exponential stability. 


\section{B. Proof of Theorem 2}

Define

$$
X=P^{-1}=\left[\begin{array}{cc}
X_{1} & 0 \\
-r_{1} X_{1} & r_{2} X_{1}
\end{array}\right] .
$$

Pre- and post-multiply $\Theta$ in (17) by $X^{T}$ and $X$, it becomes

$$
\bar{A} X+X^{T} \bar{A}^{T}+\sum_{i=1}^{n} s_{i} Q_{i}^{-1}+\sum_{i=1}^{n} s_{i} X^{T} \bar{A}_{i}^{T} Q_{i} \bar{A}_{i} X<0
$$

Let $R_{i}=Q_{i}^{-1}$ and $Y_{i}=K_{i} X_{1}, i=1, \ldots, n$. Applying Schur complement to (22) results in (11).

\section{ACKNOWLEDGMENTS}

This work is supported in part by the DFG excellence initiative research cluster Cognition for Technical Systems CoTeSys, see also www. cotesys.org, the BMBF Bernstein Center for Computational Neuroscience Munich, see also www.bccn-munich.de, the Institute for Advanced Study (IAS), Technische Universität München, see also www.tum-ias.de, and the German Research Foundation (DFG) within the Priority Programme SPP 1305 "Regelungstheorie digital vernetzter dynamischer Systeme".

\section{REFERENCES}

[1] S. Hutchison, G. Hager, and P. I. Corke, "A tutorial on visual servo control," IEEE Transactions on Robotics and Automation, vol. 12, no. 5, pp. 651-670, 1996.

[2] D. Schuurman and D. Capson, "Robust direct visual servo using network-synchronized cameras," Robotics and Automation, IEEE Transactions on, vol. 20, pp. 319-334, April 2004.

[3] P. I. Corke, High-performance visual closed-loop robot control. PhD thesis, Department of Mechanical and Manufacturing Engineering, University of Melbourne, 1994.

[4] M. Vincze, "Dynamics and system performance of visual servoing," in Proceedings of IEEE Conference on Robotics and Automation, pp. 1435, 2000.

[5] J. Hespanha, P. Naghshtabrizi, and Y. Xu, "A survey of recent results in networked control systems," Proc. of IEEE Special Issue on Technology of Networked Control Systems, vol. 95, pp. 137-162, Jan. 2007.

[6] P. Allen, B. Yoshimi, and A. Timcenko, "Real-time visual servoing," in proceedings of the IEEE International Conference on Robotics and Automation, vol. 85, pp. 1-856, Citeseer, 1991.

[7] P. Corke and M. Good, "Dynamic effects in visual closed-loop systems," IEEE Transactions on Robotics and Automation, vol. 12, no. 5, pp. 671-683, 1996.

[8] A. Ray and Y. Galevi, "Integrated communication and control systems: Part II-design considerations," Journal of Dynamic Systems, Measurements, and Control, vol. 110, pp. 374-381, 1988.

[9] E. Fridman, A. Seuret, and J.-P. Richard, "Robust sampled-data stabilization of linear systems: an input delay approach," Automatica, vol. 40, pp. 1441-1446, 2004

[10] S. Hirche, T. Matiakis, and M. Buss, "A distributed controller approach for delay-independent stability of networked control systems," Automatica, vol. 45, no. 8, pp. 1828-1836, 2009.

[11] L. Zhang, Y. Shi, T. Chen, and B. Huang, "A new method for stabilization of networked control systems with random delays," IEEE Transactions on Automatic Control, vol. 20, no. 8, pp. 117-1181, 2005.

[12] M. Sun, J. Lam, S. Xu, and Y. Zou, "Robust exponential stabilization for Markovian jump systems with mode-dependent input delay," Automatica, vol. 43, pp. 1799-1807, 2007.

[13] C.-C. Chen, S. Hirche, and M. Buss, "Controller design and experimental validation for networked control systems with time-varying random delay," Journal of the Society of Instrument and Control Engineers, vol. 47, no. 8, pp. 676-685, 2008.

[14] M. Liu, D. W. Ho, and Y. Niu, "Stabilization of markovian jump linear system over networks with random communication delay," Automatica, vol. 45, pp. 416-421, 2009.
[15] E. Marchand and F. Chaumette, "Virtual visual servoing: A framework for realtime augmented reality," in Computer Graphics Forum, vol. 21 , pp. 289-298, Wiley, 2002.

[16] D. G. Lowe, "Distinctive image fearures from scale-invariant keypoints," in International Journal of COmputer Vision, 2004.

[17] M. A. Fischer and R. C. Bolles, "Random sampled consensus: A paradigm for modeling fitting with applications to image analysis and automated cartography," in Communications of the ACM, vol. 2 , pp. 381-395, 1981.

[18] L. Deng, F. Janabi-Sharifi, and W. J. Wilson, "Stability and robustness of visual servoing methods.", in ICRA, pp. 1604-1609, IEEE, 2002.

[19] R. Kelly, R. Carelli, O. Nasisi, B. Kuchen, and F. Reyes, "Stable visual servoing of camera-in-hand robotic systems," IEEE/ASME transactions on mechatronics, vol. 5, no. 1, pp. 39-48, 2000.

[20] L. Sciavicco and B. Siciliano, Modelling and control of robot manipulators. Springer Verlag, 2000

[21] K. Åström and B. Wittenmark, Adaptive Control. Addison-Wesley, second ed., 1995.

[22] H. Gao, J. Wu, and P. Shi, "Robust sampled-data $\mathrm{H}_{\infty}$ control with stochastic sampling," Automatica, vol. 45, pp. 1729-1736, 2009.

[23] J. Löfberg, "Yalmip : A toolbox for modeling and optimization in MATLAB," in Proceedings of the CACSD Conference, (Taipei, Taiwan), 2004

[24] X. Mao, "Exponential stability of stochastic delay interval systems with markovian switching," Automatic Control, IEEE Transactions on, vol. 47, pp. 1604-1612, Oct 2002.

[25] E.-K. Boukas and Z.-K. Liu, Deterministic and Stochastic Time Delay Systems. Boston: Birkhäuser, 2002.

[26] M. S. Mahmoud and N. F. Al-Muthairi, "Design of robust controller for time-delay systems," IEEE Transactions on Automatic Control, vol. 39, pp. 995-999, 1984. 